\title{
DIE VALENZ ALS TEXTGRAMMATISCHE KATEGORIE
}

Vor mehr als dreißig Jahren kam der französische Linguist Lucien Tesnière auf die geniale Idee, die gegenseitigen Relationen zwischen dem Verb eines Satzes einerseits und den anderen Satzteilen andererseits mit den chemischen Relationen in der Struktur eines Moleküls zu vergleichen. Die Eigenschaft des Verbs, andere Wörter an sich zu binden, nannte er Verbvalenz. Tesnière schreibt (schon 1959) wörtlich: "Man kann das Verb mit einem Atom vergleichen, an dem Häkchen angebracht sind, so daß es - je nach der Anzahl der Häkchen - eine wechselnde Zahl von Aktanten an sich ziehen und in Abhängigkeit halten kann. Die Anzahl der Häkchen, die ein Verb aufweist, und dementsprechend die Zahl der Aktanten, die es regieren kann, ergibt das, was man die Valenz des Verbs nennt" (Tesnière 1980: 161). Und weiter: "Aufgrund der strukturalen Konnexionen bestehen Dependenzbeziehungen (Abhängigkeitsbeziehungen) zwischen den Wörtern. Jede Konnexion verbindet im Prinzip einen übergeordneten mit einem untergeordneten Term" (ibid., 27). Schematisch hat Tesnière diese Relationen bekanntlich in den sogenannten Stemmata (Strukturbäumen, graphischen Darstellungen von Konnexionsstrukturen; ibid., 384) wiedergegeben. Im Satz "Mon vieil ami chante cette chanson fort jolie" ist das Verb "chante" als "régissant" allen anderen Wortkonstituenten übergeordnet, wobei die untergeordneten Elemente "ami" und "chanson" in einer zweiten Rangstufe wiederum den untergeordneten Elementen "mon", "vieil", "cette", "jolie" übergeordnet sind. (Vgl. Greule 1982: 99.)

Diese Art der Satzanalyse unterscheidet sich von der binären Konstituentenanalyse, die nicht die gesamte Satzstruktur derlei übersichtlich wiederzugeben vermag. Auf der anderen Seite muß aber auch eingeschränkt werden, daß die Stemmata nicht die sukzessive Aneinanderreihung einzelner Satzbauelemente veranschaulichen können. (Vgl. ibid.)

Der Valenzbegriff erfuhr in der späteren Entwicklung viele Ausweitungen, Modifikationen und Ergänzungen, und sehr viele Linguisten (wie Heringer, Helbig, Schenkel, Engel, Schumacher, Admoni, Glinz, Brinkmann, Schwitalla) haben sich damit befaßt und befassen sich immer noch, weil gewisse Aspekte dieser Kategorie noch nicht als endgültig gelöst gelten können. Viele synonyme Bezeichnungen des Valenzbegriffs sind eingeführt worden, wie z. B. Ergänzungsbedürftigkeit, Wertigkeit, Fügungspotenz. Auch ist der Begriff ausgeweitet worden auf andere Wortarten wie das Substantiv, Adjektiv, die Präposition, außerdem ist die Valenz abgegrenzt worden von 
dem Begriff der Rektion, die nur die formale Seite der Bindung zweier Elemente meint. Einige Linguisten verfaßten sog. Valenzwörterbücher, so z. B. Helbig/Schenkel, Engel/Schumacher. Diese eignen sich ausgezeichnet für den Fremdsprachenunterricht, weil sie nicht nur quantitative und grammatische Aspekte der Verbvalenz (aber auch der Substantiv- und Adjektivvalenz) aufzeigen, sondern darüber hinaus mit der Präsentation semantischer Kompatibilitäten und Distributionsbeschränkungen in mancher Hinsicht die fehlende sprachliche Kompetenz bei Nichtmuttersprachlern ersetzen können. (Vgl. Heringer 1984: $56 \mathrm{f} ., 62$.

Das zentrale Problem bei der Verbvalenz - auf diese möchte ich mich im folgenden beschränken - war jedoch folgendes: Wie fest, wie eng binden sich einzelne Satzkonstituenten an das Verb? Die Valenztheorie unterscheidet auf dieser Grundlage notwendige Aktanten von den nicht notwendigen Angaben. (Vgl. Greule 1982: 104, 287.) Notwendige Aktanten sind demnach jene Wörter, oder besser Satzglieder, die im Stellenplan des Verbs enthalten sind und auch aufgrund der Sememstruktur (vgl. Sommerfeldt 1991: 25) des Verbs erwartbar sind. So kann man z. B. sagen, daß das Verb wohnen die Angabe des Ortes erfordert:

1. Er wohnt in einem Hochhaus.

Diese Ortsangabe ist kompatibel mit der semantischen Beschaffenheit des Verbs. Das Ausbleiben dieser Ortsangabe führt im Normalfall zu einer defekten Satzstruktur.

2. Er wohnt ...

Das Verb wohnen ist also ein zweiwertiges Verb, neben dem Subjekt, das sogar bei unpersönlichen Verben (dort zwar in Form des unpersönlichen Pronomens "es") obligatorisch steht, muß im struktural, inhaltlich und intonatorisch vollständigen deutschen Satz, der dieses Verb enthält, noch eine weitere Größe stehen, und zwar der Lokativ.

Auf der anderen Seite ist es aber auch möglich, daß ein Attribut, das grammatisch-strukturell völlig weglaßbar ist, in kommunikativer Hinsicht obligatorisch sein kann, weil es eine Fokussierung mit sich bringt:

3. Die Henne legt große(!) Eier. (Heringer 1984: 38.)

Auch Angaben können in einem Text kommunikativ notwendiger sein als Ergänzungen:

4.1. Der Junge lebte noch(!) gestern(!).

4.2. Den Fehler hatte er zu spät(!) entdeckt.

4.3. Arbeitet Fritz in Leipzig? Ja, er arbeitet dort(!). (Nikula 1986: 265 f.)

"Kommunikativ notwendige Konstituenten sind Elemente, die in einem Text nicht ausgelassen werden können, wobei sowohl strukturell notwendige (Ergänzungen) als auch strukturell nicht notwendige Konstituenten (Angaben) kommunikativ notwendig sein können" (ibid.). 
Auch Helbig erwähnt im Zusammenhang mit der Notwendigkeit, d. h. Obligatorität von Satzgliedern, daß es nicht auf die satzsyntaktische (d. h. valenzgebundene Ergänzungen im bisherigen Sinne) ankomme, sondern vielmehr auf die t e x $\mathrm{t}$ s y n t a k i s che (gesperrt im Original) (Thema-Rhema-Gliederung) oder kommunikative Notwendigkeit (bestimmte Partikeln sind morphosyntaktisch freie Angaben, illokutiv jedoch obligatorisch) (1985: 153). "Es besteht kein Zweifel daran, daß es in der Tat keine direkte Entsprechung zwischen satzsyntaktischer, textsyntaktischer und kommunikativer Notwendigkeit gibt, daß also auch (oder gerade) freie Angaben im Sinne der syntaktischen Valenz rhematische Glieder und deshalb vom Informationsgehalt her unverzichtbar sein können" (ibid., 154). Schwitalla teilt diese Auffassung und argumentiert sie folgendermaßen: "Es ist ja unbestritten, daß das kommunikative Gewicht einer Satzäußerung gerade auf den Angaben liegen kann, und daß die Variabilität und die relativ freie Hinzufügbarkeit von Angaben den unendlich vielen Mitteilungsabsichten in konkreten Situationen gerecht werden können" (1985: 268).

Die Valenztheorie hat verschiedene Mehtoden und Tests eingeführt, mit denen man einzelne Elemente im Satz auf ihre Notwendigkeit bzw. Weglaßbarkeit prüfen kann. Mit Hilfe des Eliminierungstests kann man Satzglieder im Satz so lange tilgen, bis der Satz noch verständlich, noch akzeptabel ist. Nikula betont, daß mit einem Eliminierungstest eigentlich die kommunikative Notwendigkeit eines Elements getestet wird (1986: 266).

Hier gelangen wir an den Punkt, an dem unbedingt zwei Aspekte der Valenztheorie hervorzuheben sind. Erstens: Verschiedene Transformationsproben wotrden lange Zeit nur auf der Satzebene durchgeführt. Zweitens: Von der strukturellen, d. $h$. grammatischen Valenz ist unbedingt noch eine semantische und von dieser eine pragmatische Valenz zu unterscheiden.

Der erste Aspekt bedeutet, daß man die Notwendigkeit einzelner Satzkonstituenten bislang "extrakommunikativ" (Heringer 1984: 37) betrachtet hat, d. h., zur Bestimmung der Minimalstruktur einer Satzäußerung hat man verschiedene Kontextsituationen ausund hinzugedacht. Auf diesem Weg ist man zu der Erkenntnis gekommen, daß die Valenz nicht auf den Satz beschränkt werden kann, sondern daß sie unbedingt auf der Textebene beobachtet werden muß.

Der zweite Aspekt (semantische und kommunikative Valenz) ist aufs engste mit dem ersten verknüpft: Nicht nur grammatisch und semantisch (vgl. Helbig 1985: 154) ausgerichtet ist die Kategorie der Verbvalenz, sondern in erster Linie pragmatischkommunikativ. (Vgl. Sommerfeldt 1991: 22, Heringer 1984: 34, Nikula 1986: 267; Schwitalla hingegen warnt davor, allgemein von einer pragmatischen Valenz zu sprechen (1988: 80).) Pragmatische Regularitäten überlagern die grammatischen und semantischen, d. h. mit anderen Worten, auf der Satzebene kann gegen grammatische, ja auch semantische Normen verstoßen werden, wenn die Kohärenz des Rahmentextes, seine thematische Zusammengehörigkeit, es als möglich oder sogar notwendig 
voraussetzt. (Das Zimmer lacht.) Wenn ich also von einem verständlichen, noch akzeptablen Satz rede, dann meine ich dies textgrammatisch-pragmatisch.

Aus der Textperspektive gesehen, kommen jene pragmatischen Faktoren viel besser zum Vorschein, die mitbestimmen, was an der Oberfläche eines Textes weglaßbar ist. In erster Linie denke ich dabei mit Heringer (1984: 39) an den gemeinsamen Wissensrahmen, der mit dem Text abgesteckt ist und der auf der bloßen Satzebene nur sehr beschränkt gegeben werden kann. Der Text als Ganzes liefert auch zusätzliche Informationen darüber, ob zu diesem gemeinsamen Wissensrahmen auch frames und scripts (tradierte Wissensschemata) (vgl. Heringer 1984: 48) zuzurechnen sind, die wiederum die Verständigung auf ihre Art lenken. Aber auch das Vorhandensein des globalen enzyklopädischen Hintergrundwissens und dessen Ausnutzung zeichnet sich erst auf der Textebene ab. Nikula schreibt in diesem Zusammenhang, daß "die Valenz nicht nur die Struktur der Sätze stark beeinflußt, sondern auch zur Gestaltung von Texten beiträgt, und zwar sowohl strukturell als auch kommunikativ-pragmatisch. Die Rolle der Valenz als Vermittlerin zwischen Lexikon und Text, zwischen Struktur und kommunikativer Funktion, erklärt einerseits die Schwierigkeiten ihrer Beschreibung, zeigt aber andererseits, daß weitere Untersuchungen der Beziehungen zwischen Valenz und Text nicht nur theoretisch notwendig sind, sondern auch für die Praxis offenbar wertvoll sein werden" (1986: 268).

In grammatischer Hinsicht bestimmt die Verbvalenz im voraus die Struktur des Satzes. Heringer veranschaulicht dies mit dem folgenden Vergleich: "Ein Verb, das ist so, wie wenn man im dunklen Raum das Licht anknipst. Mit einem Schlag ist eine Szene da" (1984: 49). Bekannt ist auch der Theater-Vergleich von Tesnière: Dem Prozeß des Dramas entspricht das Verb, den "acteurs" die Aktanten und den "circonstances" die Zirkonstanten. (Aus Greule 1982: 103.)

In der Performanz kommt es immer wieder zu unvollständigen Satzkonstruktionen. Weinrich spricht dabei von der Unterwertigkeit bzw. von der Valenz-Minderung (1993: 136). Allein auf der Satzebene sind Kontraktionserscheinungen beobachtbar, die sprachökonomisch $\mathrm{zu}$ erklären sind, jedoch ihrerseits der Erfüllung gewisser Bedingungen unterliegen. So wird z. B. im zusammengezogenen Satz

5. Er studierte in Jena und seine Schwester in Berlin.

das Subjekt des ersten Satzes, weil identisch, nicht wiederholt. (Vgl. Helbig/Buscha 1993: 650 f.) Auch in Infinitivkonstruktionen bleiben identische Satzglieder aus demselben Grund aus:

6. Nach der erfolgreich durchgeführten Operation hofften die Ärzte, seine Leistungsfähigkeit wesentlich erhöhen zu können.

Komplizierter als auf der Satzebene scheinen Auslassungen auf der Textebene. Texte sind bekanntlich komplexe Strukturen, die in der Regel aus mehreren Sätzen bestehen. Für Texte ist es typisch, daß sie relativ abgeschlossene kommunikativinformative Einheiten darstellen (ihre Kohärenz). (Vgl. Sommerfeldt 1991: 21). Das 
formale Kennzeichen dieser thematischen Zusammengehörigkeit ist an der Oberfläche die Kohäsion, die Verwobenheit, das Verflochtensein von aneinandergereihten Sätzen durch die Wiederaufnahme einzelner propositionaler Elemente durch Synonyme, Pronomina (Pronominalisierung, Semrekurrenz), durch den Artikelgebrauch sowie durch die temporale Einheitlichkeit. An der Textoberfläche kann es dazu kommen, daß gewisse Leerstellen des Verbs, aber auch des Substantivs, nicht besetzt sind. Was in einem Satz fehlt, taucht manchmal im darauffolgenden oder texttopologisch noch weiter entfernten Satz auf, so daß der Text als Ganzes erst recht allen Bedingungen der strukturellen und pragmatischen Valenz einzelner Sätze Genüge leistet. (Vgl. Giora 1983.) Schauen wir uns das genauer im folgenden, einer Abhandlung von Sommerfeldt (1991: 26) entnommenen Text an:

\section{Skinheads 1 verletzen 2 in Frankreich6 acht Menschen}

1. In den nordfranzösischen Städten Rouen und Brest 6 haben randalierende Skinheads1 am Wochenende 7 acht Menschen schwer verletzt 2 . 2 . 24 Jugendliche 1 ... wurden am Montag 7 wegen vorsätzlicher Körperverletzung 2 und Waffenbesitz 3 unter Anklage gestellt4. 3. Die mit Baseballschlägern und Schlagringen bewaffneten 3 Randalierer 1 gehören zu etwa 200 Skinheads 1 aus Frankreich 6 und anderen Ländern6, die zu einem Rockkonzert5 nach Brest6 gezogen waren. 4. Da die Stadtverwaltung 4 weitere Ausschreitungen 2,3 befürchtete, wurde die Veranstaltungs abgesagt 4 .

"Randalierende Skinheads" aus dem 1. Satz (und teilweise dem Titel) werden im 2. Satz durch "24 Jugendliche", im 3. Satz durch "Randalierer" und "200 Skinheads" (Teil-Ganzes-Relation) wiederaufgenommen. "Schwer verletzt" aus dem 1. Satz (und teilweise dem Titel) wird im 2. Satz durch "Körperverletzung" präzisiert und durch "weitere Ausschreitungen" im 4. Satz abgerundet. Hierzu gehört auch die Kontiguitätsbeziehung "Waffenbesitz" (2. Satz). "Am Wochenende" aus dem 1. Satz wird vermittels einer semantischen Kontiguität in der präpositionalen Fügung "am Montag" im 2. Satz weitergeführt. "Waffenbesitz" in Satz 2 wird im darauffolgenden Satz 3 durch "Baseballschläger/n/ und Schlagringe" fortgesetzt. "Unter Anklage gestellt" (Satz 2) wiederholt sich durch Semrekurrenz (Gerichtswesen) in der Formulierung "die Veranstaltung absagen" (Satz 4). "Rouen und Brest" aus Satz 1 ist im 3. Satz in "Brest" wiederzufinden (merke auch "Frankreich" im Titel), und "Rockkonzert" im 3. Satz wird in der "Veranstaltung" im 4. Satz synsemantisch wiederaufgenommen. Die bestimmten Artikel im 3. Satz ("Die ... Randalierer") und im 4. Satz ("die Stadtverwaltung", "die Veranstaltung") signalisieren ebenfalls die aus dem Vortext bereits bekannten bzw. erwähnten Größen. In diesem Kurztext wird als Erzähltempus einheitlich das Präteritum verwendet.

Neben den isotopischen (vgl. Sommerfeldt 1991: 22) Verknüpfungen und anderen Kohärenzmitteln sind im obigen Text weitere Besonderheiten festzustellen. Nach Sommerfeldt ist die Leerstelle des Instruments ("mit Baseballschlägern und Schlagringen") im 1. Satz nicht besetzt. Die Instrumentalbestimmung ist hier zwar keine notwendige Ergänzung des Verbs "verletzen", sie ist aber auch keine freie 
Angabe, sondern sie bildet eine Peripherieerscheinung der Valenz dieses Verbs (vgl. Sommerfeldt 1991: 24 und Heringer 1984: 45 f.). Die Instrumentalbestimmung wird durch die Bedeutung des Verbs "verletzen" präsupponiert und erst im 3. Satz aktiviert ("mit Baseballschlägern und Schlagringen"). Somit ist der 3. Satz auf eine indirekte Art mit dem ersten verzahnt. Im 2. Satz ist die Leerstelle des Agens ("Rechtsanwalt der Stadtverwaltung von Rouen und Brest") nicht besetzt. Sie ergibt sich jedoch aus dem Sachwissen (Gerichtswesen) und aus den im 1. Satz enthaltenen Ortsbezeichnungen ("Rouen", "Brest"). Weiterhin ist im 4. Satz die Leerstelle des Substantivs "Stadtverwaltung", die die Zugehörigkeit kennzeichnet, nicht besetzt. Wir können jedoch aus dem logischen Zusammenhang im Text schlußfolgern, daß es sich um die Stadtverwaltung von Rouen handelt. Daher auch der die Referenzidentität signalisierende bestimmte Artikel "die". Auch die Leerstelle des Agens im Hauptsatz des 4. Satz ist nicht explizit genannt, denn sie ist identisch mit dem Subjekt im vorangestellten Nebensatz, und sie kann ausgefüllt werden unter Hinzuziehung der Ortsangaben im ersten Satz.

Wir sehen, daß diese definiten Auslassungen von Aktanten (vgl. Nikula 1986: 264 und Schwitalla 1988: 74) in verschiedenen Sätzen des obigen Textes über die üblichen textphorischen Mittel hinweg, wie sie oben von mir demonstriert worden sind, zusätzliche Kohäsionsmittel darstellen. Die fehlenden Leerstellen bewirken, daß einzelne Sätze noch enger voneinander abhängig, noch fester miteinander verbunden sind. Der Rezipient ist dadurch gezwungen, sich mehr oder weniger unbewußt noch stärker dem Kontext widmen zu müssen (vgl. Sommerfeldt 1991: 29 und Giora 1983), um diesem Kontext fehlende Informationen zu entnehmen. Von ihm wird größere Konzentration vorausgesetzt, die Nichterfüllung dieser Bedingung führt zu Schwierigkeiten in der Textrezeption. Und so wie bei anderen Kohäsionsmitteln erfolgt die Besetzung der Leerstellen in benachbarten Sätzen. "Es kann /aber/ auch Beziehungen geben, die sich über mehrere Sätze, ja über umfangreichere Texte erstrecken" (Sommerfeldt 1991: 29). "/E/ine nicht besetzte Leerstelle /ist/ als eine Anweisung zu betrachten /.../, nach einer "Füllung" zu suchen, d. h. die Auslassung (bzw. die Nicht-Besetzung) hinterläßt immer eine "Spur"" (Nikula 1986: 265). Das Eliminieren von Satzgliedern hat also zur Folge, "daß durch die Herstellung kontextualer Beziehungen die Einheit des Textes unterstrichen wird" (Sommerfeldt 1991: 24).

Noch auffälliger ist diese Erscheinung in dialogischen Texten. (Vgl. Michajlow 1971.) Diese unterscheiden sich von den monologischen hauptsächlich durch zwei Charakteristika:

1. In ihnen haben wir keine lineare Satzreihe, sondern eine auf der Minimaldialogsequenz Frage-Antwort basierende alternierende Satzreihe;

2. Eine dem Sprecher und Hörer (sie wechseln aber in der Regel ständig ihre Rollen) gemeinsame kommunikative Situation liefert eine Menge Informationen, die nicht versprachlicht werden, ohne daß der Diskurs für die daran Beteiligten dadurch 
unverständlich wird. Anders ist es dagegen mit den Außenstehenden. Danielle Laroche-Bouvy schreibt: "La transcription écrite d'une conversation ou même d'une interview animée peut être parfaitement illisible, incompréhensible sans le secours de l'enrigstrement" (1992: 88). Die Auslassungen von notwendigen Aktanten erfolgen somit nach anderen Regularitäten, die der Hauptbedingung unterliegen, daß die Verständigung zwischen den Kommunikationspartnern nicht beeinträchtigt wird. (Vgl. jedoch Schwitalla 1988: 80.)

Ich führe im weiteren einige Belege an, die typisch für den dialogischen Text sein könnten:

\subsection{Können wir anfangen? (mit der Diskussion)}

8.2. Hier möcht' ich widersprechen. (dem Vorredner)

8.3. Ich möchte schließen mit der Feststellung. (den Beitrag)

8.4. Darf ich mal unterbrechen? (den Redner)

8.5. Ich bin noch nicht fertig! (mit meinem Vortrag)

An dieser Stelle sei auch erwähnt, daß die gesprochene (machmal auch die geschriebene) Umgangssprache von der Möglichkeit Gebrauch macht, das Vorfeld leer zu lassen. (Siehe bei Weinrich 1993: 78.) Aus diesem Sprachgebrauch haben sich viele stereotype Ausdrücke entwickelt, mit denen man in mündlicher Wechselrede auf eine vorher geäußerte Meinung, Frage, Aufforderung reagiert:

hast ja recht stimmt ja gar nicht

ist schon klar kommt gar nicht in Frage

Die Anknüpfung an den voraufgehenden Kontext, die in schriftlichen Texten hauptsächlich dem Vorfeld obliegt, kann unterbleiben, weil die lebhafte Wechselrede durch eine starke Situationsbindung gekennzeichnet ist, die die Strategien der Vertextung von schriftlichen Texten stark modifiziert.

Vor dem Hintergrund der bisherigen Überlegungen zur Valenzreduzierung wage ich die Aufstellung der folgenden Hypothese: das aus einer Situation resultierende Quantum des gemeinsamen Wissens der Kommunikationspartner ist indirekt proportional mit der Menge der verwendeten sprachalichen Mittel zur Erreichung eines kommunikativen Ziels. Unbedingt ist dabei auch der Parameter der OffizialitätNichtoffizialität (vgl. Helbig 1985: 154, Schwitalla 1988: 77) - d. h. der Kommunikationssphäre, des Tätigkeitsbereichs - zu beachten. Diese Hypothese ist zu überprüfen und u. U. zu verifizieren, indem man dialogische Texte zu einem bestimmten Thema in monologische mit demselben Thema transponiert.

In dialogischen Texten kommen konzentriert auch Stilfiguren vor, deren Wirkung auf die Reduzierungen der Valenzverhältnisse zurückzuführen ist. Das sind außer der Ellipse, von der in extenso oben gesprochen wurde, noch folgende abweichende Satzkonstruktionen: die Aposiopese, das Anakoluth und die Isolierung (vgl. Fleischer/Michel 1977: 179 ff.). 
Die Aposiopese als ein formaler und inhaltlicher plötzlicher Abbruch eines begonnenen Satzes, dem meistens ein neuer Satz folgt:

9. Es gab bestimmt einen Zusammenhang zwischen ihm und Höfel. Wenn man ihn nur wüßte, dann könnte man das Kind vielleicht ... Diese verdammte Heimlichtuerei des Bochow ... Blind und unwissend ließ er ihn. (Apitz, Nackt unter Wölfen)

Das Anakoluth als eine folgewidrige Satzfügung, ein Übergang aus einer begonnenen Konstruktion in eine andere:

10. Das habe ich wohl ungefähr, haben meine Eltern wohl, ungefähr drei, vier Jahre war ich wohl, da sind wir nach K. K. gezogen.

Die Isolierung zeigt bekanntlich eine enge Verbindung zum vorhergehenden Satz. Ein Satzglied wird im neuen Satz verselbständigt:

11. Auf Wiedersehen, sagte Winkelfried. Nicht verärgert, nur gleichgültig. (Seghers, Das Duell)

Das sind Erscheinungen, die mit der sukzessiven Denkweise beim spontanen Sprechen einhergehen. Wenn sie dagegen in geschriebener Sprache vorkommen, werden sie gekonnt als Stilmittel eingesetzt, um in stilisierten Dialogen - aber auch in monologischen Autortexten - das dynamische Gepräge des Gesprochenen nachzuahmen. Diesen Stilfiguren liegt eine teilweise oder komplette Reduktion bzw. Modifikation (Valenzminderung, Valenzerhöhung) grundlegender Valenzverhältnisse zwischen dem Valenzträger Verb und anderen Aktanten zugrunde.

Abschließend sei noch im Bereich der Funktionsverbfügungen auf die Valenzänderung des Typs "beeindrucken jn." - "(einen guten) Eindruck machen (auf jn.)", "lauern auf jn." - "auf der Lauer liegen" hingewiesen, wo intratextuelle Relationen die Verwendung von Strukturen ermöglichen, die valenzmäßig als Reduktionen aufzufassen sind.

Wir sehen, daß diese Erscheinungen auch ausgprägte stilistische Funktionen ausüben, daß daher bei ihrer Erforschung unbedingt auch der stilistische Aspekt herangezogen werden muß. Es handelt sich offenbar um eine vielschichtige Problematik, deren Komplexität noch auszuloten ist. Lucien Tesnière hat mit seinen Untersuchungen dazu einen bahnbrechenden Beitrag geleistet.

\section{Bibliographie:}

Bračič, Stojan (1993): Kommunikative Funktion der gegenwärtigen deutschen Umgangssprache in Pressereiseerzählungen. Frankfurt am Main, Berlin, Bern, New York, Paris, Wien.

Engel, Ulrich/Schumacher, Helmut (1978): Kleines Valenzlexikon deutscher Verben. Tübingen. 
Fleischer, Wolfgang/Michel, Georg (1977): Stilistik der deutschen Gegenwartssprache. Leipzig.

Giora, Rachel (1983): Segmentation and segment cohesion: On the thematic organization of the text. In: Text $3 / 2$, S. 155-181.

Greule, Albrecht (1982): Valenz, Satz und Text. Syntaktische Untersuchungen zum Evangelienbuch Otfrieds von Weißenburg auf der Grundlage des Codex Vindobonensis. München.

Helbig, Gerhard (1985): Valenz und Kommunikation. In: DaF, 3/22, S. 153-156.

Helbig, Gerhard/Buscha, Joachim (1993): Deutsche Grammatik. Ein Handbuch für den Ausländerunterricht. Leipzig, Berlin, München, Wien, Zürich, New York.

Helbig, Gerhard/Schenkel, Wolfgang (1982): Wörterbuch zur Valenz und Distribution deutscher Verben. Leipzig.

Heringer, Hans Jürgen (1984): Neues von der Verbszene. In: Gerhard Stickel (Hg.): Pragmatik in der Grammatik. Sprache der Gegenwart 60. Düsseldorf. S. 34-64.

Laroche-Bouvy, Danielle (1992): Dialogue écrit, dialogue spontané. In: Stati, Sorin/ Edda Weigand (Hg.): Methodologie der Dialoganalyse. Tübingen. S. 87-96.

Michajlow, Leonid (1971): Zu Fragen der Reduzierung der Valenz im Dialog. In: DaF, 3/8, S. 180-182.

Nikula, Henrik (1986): Valenz und Text. In: DaF, 5/23, S. 263-268.

Schwitalla, Johannes (1985): Verbvalenz und Text. In: DaF, 5/22, S. 266-270.

Schwitalla, Johannes (1988): Kommunikative Bedingungen für Ergänzungsrealisierungen. In: Linguistische Studien, Reihe A. Berlin. S. 74-85.

Sommerfeldt, Karl-Ernst (1991): Valenz und Textkohärenz. In: Beiträge zur Erforschung der deutschen Sprache 10. Tübingen. S. 21-29.

Tesnière, Lucien (1980): Grundzüge der strukturalen Syntax. Hg. und übers. v. Ulrich Engel. Stuttgart.

Weinrich, Harald (1993): Textgrammatik der deutschen Sprache. Mannheim, Leipzig, Wien, Zürich.

Povzetek

VALENCA KOT KATEGORIJA BESEDILNE SLOVNICE

Tesnièrjeva zasluga je, da je pred več kot tridesetimi leti prenesel pojem valence iz naravoslovja v jezikoslovje. Valenco je označil kot lastnost glagola, da v stavku veže nase enega ali več delovalnikov.

Temeljni problem, ki se pri tem odpira, je v tem, kako močno se posamezni stavčni členi vežejo na glagol. Razlikovati je med skladenjsko valenco na eni strani ter pomensko in pragmatično valenco na drugi strani. Valence glagola se namreč ne da opredeliti zgolj na povedni ravni, temveč jo je mogoče pravilno določiti šele v besedilu kot celoti. Tako se valenca izkaže kot parameter z besedilotvorno relevanco. Določeni stavčni členi so skladenjsko tesno vezani na glagol, ker jih sememska struktura glagola obvezno predvideva; ravno ti stavčni členi pa lahko $v$ površinski strukturi izostanejo. Po drugi strani pa posamezni členki in prilastki z ničto skladenjsko odvisnostjo od glagola lahko dobijo pragmatično težo in niso izpustljivi. Če so delovalniki, ki jih na podlagi glagolske valence pričakujemo, izpuščeni, zahteva to posebno koncentracijo recipienta. Le-ta mora namreč iz besedila kot celote izluščiti delovalnike, ki se včasih eksplicitno pojavijo šele v nadaljnjih povedih besedila, včasih pa sploh ne. 